\title{
Nonparametric estimation of a dependent competing risks model for unemployment durations
}

\author{
Gerard J. van den Berg • \\ A. Gijsbert C. van Lomwel • Jan C. van Ours
}

Received: 15 February 2005 / Accepted: 15 December 2006 / Published online: 17 March 2007

(C) Springer-Verlag 2007

\begin{abstract}
In this paper we simultaneously analyze transitions from unemployment to employment and to nonparticipation. We estimate a dependent competing risks model with nonparametric specifications of the destination-specific duration dependence and unobserved heterogeneity terms, allowing for mutual dependence of the unobserved heterogeneity terms. We use an administrative data set covering all registered French unemployed over the period 1988-1994, stratified by gender type, duration class and exit state.
\end{abstract}

Keywords Exit rate Hazard - Unobserved heterogeneity ·

Duration dependence $\cdot$ Nonparticipation

JEL Classification J64 · C41

We thank the Editor and three anonymous referees for their useful comments. A preliminary version of this paper was distributed under the title "Individual variation in exit rates from unemployment: a nonparametric multivariate analysis using aggregate data". The Département de Marché du Travail of the Ministère du Travail, de l'Emploi et de la Formation Professionelle of France kindly provided the data.

G. J. van den Berg $(\bowtie)$

Department of Economics, Free University Amsterdam, IFAU, IFS, IZA, CEPR,

De Boelelaan 1105, NL-1081 HV Amsterdam, The Netherlands

e-mail: gjvdberg@xs4all.nl

A. G. C. van Lomwel

Social Security Division, Achmea Insurance Company, P.O.Box 700,

NL-7300 HC Apeldoorn, The Netherlands

J. C. van Ours

Department of Economics, Center for Economic Research, CEPR, IZA,

Tilburg University, P.O. Box 90153, NL-5000 LE Tilburg, The Netherlands 


\section{Introduction}

Studies on unemployment durations often focus on whether the individual transition rate to employment decreases as a function of the elapsed duration. To examine this one has to correct for the presence of unobserved heterogeneity. Negative duration dependence at the individual level and unobserved heterogeneity both lead to negative duration dependence of the observed transition rate, but they have different policy implications. Negative duration dependence implies that emphasis should be put on the prevention of long-term unemployment. See for example Manning and Machin (1999), who emphasize the usefulness of policies aimed at intervening long before individuals have become long-term unemployed. This type of policy, however, will not be optimal if unobserved heterogeneity is the cause of negative duration dependence of the observed transition rate. In that case, policy should be aimed at screening of newly unemployed.

To study these issues one also has to take into account that individuals may make transitions to other states than employment. Notably, they may move into nonparticipation (or "out of the labor force"). If one lumps transitions to employment and nonparticipation together, and if these transitions have different determinants, then this may lead to incorrect inference concerning the transition rate to work. Moreover, if one treats transitions to nonparticipation as right-censoring of the duration until work, and if the unobserved determinants of the transition rates to nonparticipation and work are mutually dependent, then the right-censoring is dependent, and standard duration analysis leads to incorrect inference. ${ }^{1}$

In this paper we estimate a model with separate transitions to nonparticipation and work, allowing both to depend on unobserved heterogeneity terms that can be mutually dependent themselves. This is a dependent competing risks model in which the individual destination-specific transition probabilities (or "exit probabilities") have mixed proportional hazard (MPH) structures (see Lancaster 1990; Van den Berg 2001 for overviews). In continuous time, such models are identified without the need to impose parametric functional form assumptions on the duration dependence terms or the bivariate distribution of the two unobserved heterogeneity terms (Heckman and Honoré 1989; Abbring and Van den Berg 2003). The main identifying assumption is basically that the two exit rates should not vary with the observed covariates in exactly the same way.

We use a discrete MPH model because it is a convenient way to express the observed exit probabilities as the product of calendar time effects, the genuine duration dependence effect, and the expected value of the heterogeneity term conditional on survival. Note that the model we use should not be interpreted as an approximation to the continuous time MPH model. Rather, it should be

\footnotetext{
1 Inference is also incorrect if one lumps the states of unemployment and nonparticipation together; Flinn and Heckman (1983) show that the transition rates to work differ between unemployment and nonparticipation.
} 
regarded as a flexible accounting device for discrete aggregate duration data, with an appealing interpretation. As shown in Abbring et al. (2002) and in the present paper, the identification of our type of model and continuous-time MPH models is established along similar lines. Our model specification can be derived as a time-aggregated version of an underlying continuous-time model, but the latter is somewhat different from the continuous-time MPH model.

The model and estimation method we developed need discrete-time timeseries data on aggregate numbers of individuals in different unemployment duration classes. Such aggregate data can be used to calculate outflow from different duration classes for each calendar time point. The main disadvantage of aggregate data compared to micro data is the lack of detail. There are also clear advantages related to the use of aggregate data. An important advantage is that they cover a much longer time span than is usual in micro data. Another major advantage is that usually they do not suffer from attrition due to nonresponse which can be a serious problem, since attrition may be induced by the occurrence of a transition. Of course, also with aggregate data there may be attrition, but since the collection of administrative data is institutionalized it will be substantially less than in the case of survey data that rely on individuals responding. Also, aggregate data have the advantage that they provide the exact values of the exit probabilities out of the different duration classes considered (averaged over unobserved heterogeneity). Finally, although it is true that high quality micro data become rapidly available in many countries this is not true for all countries and usually the micro datasets do not go far back in time. Analysis of aggregate unemployment dynamics can generate complementary insights derived from micro studies.

As an example to illustrate our method, we use rather unique French administrative data over the period 1988-1994, covering all registered French unemployed. These data are quarterly and cover individuals who are looking for full time permanent jobs. Because of the discrete nature of the data we specify and estimate a discrete time model. The main explanatory covariate is calendar time, capturing business cycle and seasonal effects. The model and the nonparametric estimation method that we develop and apply extend those in Van den Berg and Van Ours (1996) to a competing risks setting. The way in which the dependence between the unobserved heterogeneity terms is estimated provides intuition behind the identification of dependent competing risks models in general.

The outline is as follows. In the next section, the model and estimation method are introduced. We describe our data in Sect. 3. Here, we also show how the exit probabilities are constructed from the data. Estimation results are reported in Sect. 4. Concluding remarks are made in Sect. 5.

\section{The model and the estimation method}

\subsection{The model}

A spell of unemployment can end with an exit to employment (E) or to nonparticipation $(\mathrm{N})$. We assume that at the individual level, the two conditional 


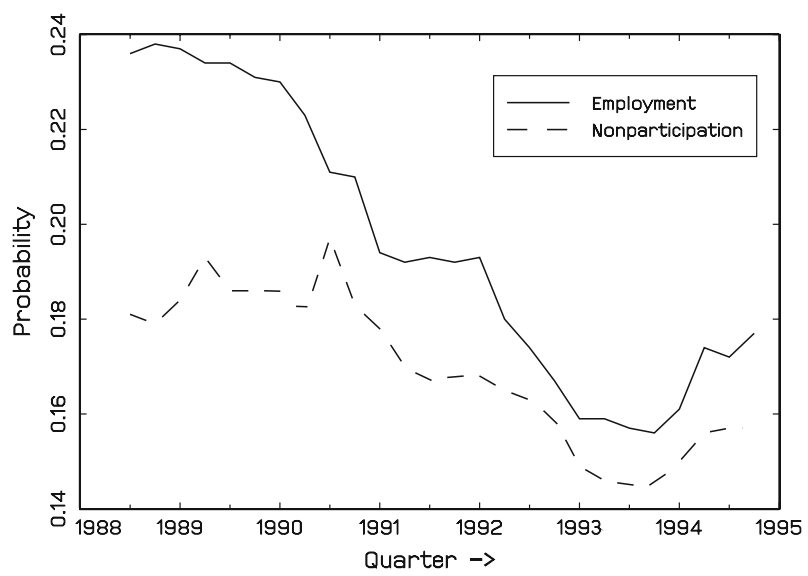

Fig. 1 Calendar time variation in exit probabilities during first quarter

exit probabilities satisfy MPH-type specifications (see e.g., Van den Berg and Van Ours 1996; Abbring et al. 2002). We only have two observed explanatory variables: the gender of the individual and calendar time. We estimate the models separately for both gender types, and therefore suppress the conditioning on the gender type. The (conditional) exit probability to destination $i$ after $t$ periods of unemployment, given the current value of calendar time $\tau$, and given the unobserved heterogeneity term $v_{i}$ that affects the exit to destination $i,(i=E, N)$, is now specified as

$$
\theta_{i}\left(t \mid \tau, v_{i}\right)=\psi_{1 i}(t) \psi_{2 i}(\tau) v_{i}
$$

The individual over-all exit probability out of unemployment is defined as $\sum_{i} \theta_{i}\left(t \mid \tau, v_{i}\right)$, so that we require - by assumption - the model determinants to satisfy the inequality $0<\sum_{i} \theta_{i}\left(t \mid \tau, v_{i}\right)<1$. $^{2}$ The functions $\psi_{1 i}$ and $\psi_{2 i}$ represent the effect of unemployment duration and calendar time. The unobserved heterogeneity terms $v_{E}$ and $v_{N}$ are allowed to be correlated. We assume that the distribution $G$ of $\left(v_{E}, v_{N}\right)$ among the inflow, and the individual realizations $v_{i}$, do not change over time. This implies that the individual exit probabilities do not depend on the moment of entry.

\subsection{Empirical implementation}

We are primarily interested in estimating the duration dependence functions and the unobserved heterogeneity distribution. As will be explained in Sect. 3, the data provide destination-specific exit probabilities at calendar times $\tau$ and at durations $t$ (we take $t$ and $\tau$ to have the same measurement scale, apart from the difference in origin). These probabilities (or "crude hazards") $\theta_{i}(t \mid \tau)$ are of

\footnotetext{
2 Empirically this issue is not problematic, because the sum of the exit probabilities is substantially smaller than one, see Figs. 1 and 2.
} 


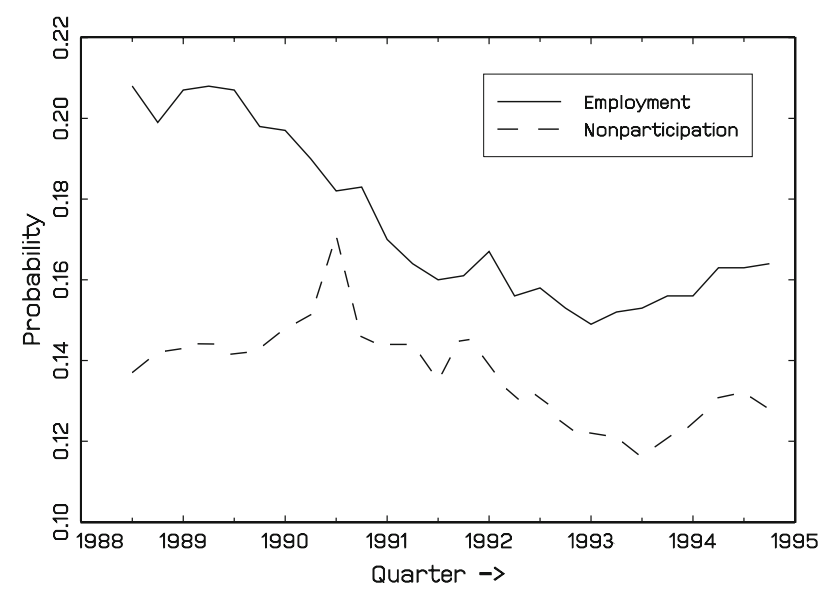

Fig. 2 Calendar time variation in exit probabilities during second quarter

course aggregated over unobserved heterogeneity. To express them in terms of the model determinants, we have to integrate over $v_{E}, v_{N}$. Consider $\theta_{i}(t \mid \tau)$ for $t=0, i=E, N$;

$$
\begin{aligned}
\theta_{i}(0 \mid \tau) & =E_{v_{i}}\left[\psi_{1 i}(0) \psi_{2 i}(\tau) v_{i}\right] \\
& =\psi_{1 i}(0) \psi_{2 i}(\tau) \mu_{1 i},
\end{aligned}
$$

where

$$
\mu_{k i}:=E\left[v_{i}^{k}\right], \quad k=1,2 .
$$

For $t=1, i \neq j=E, N$ we get

$$
\begin{aligned}
& \operatorname{Pr}(t=1 \wedge i \mid \tau) \\
& \quad=E_{v_{i}, v_{j}}\left[\left(1-\psi_{1 i}(0) \psi_{2 i}(\tau-1) v_{i}-\psi_{1 j}(0) \psi_{2 j}(\tau-1) v_{j}\right) \cdot \psi_{1 i}(1) \psi_{2 i}(\tau) v_{i}\right] \\
& \quad=\psi_{1 i}(1) \psi_{2 i}(\tau) \cdot E_{v_{i}, v_{j}}\left[v_{i}-\psi_{1 i}(0) \psi_{2 i}(\tau-1) v_{i}^{2}-\psi_{1 j}(0) \psi_{2 j}(\tau-1) v_{i} v_{j}\right] \\
& \quad=\psi_{1 i}(1) \psi_{2 i}(\tau) \cdot\left[\mu_{1 i}-\psi_{1 i}(0) \psi_{2 i}(\tau-1) \mu_{2 i}-\psi_{1 j}(0) \psi_{2 j}(\tau-1) \mu_{1 i 1 j}\right],
\end{aligned}
$$

where

$$
\mu_{1 i 1 j}:=E\left[v_{i} v_{j}\right]=\operatorname{Cov}\left(v_{i}, v_{j}\right)+\mu_{1 i} \mu_{1 j}
$$

so that

$$
\begin{aligned}
\theta_{i}(1 \mid \tau) & =\frac{\operatorname{Pr}(t=1 \wedge i \mid \tau)}{\operatorname{Pr}(t \geq 1 \mid \tau)} \\
& =\psi_{1 i}(1) \psi_{2 i}(\tau) \cdot \frac{\mu_{1 i}-\theta_{i}(0 \mid \tau-1) \frac{\mu_{2 i}}{\mu_{1 i}}-\theta_{j}(0 \mid \tau-1) \frac{\mu_{1 i 1 j}}{\mu_{1 j}}}{1-\theta_{i}(0 \mid \tau-1)-\theta_{j}(0 \mid \tau-1)}
\end{aligned}
$$

We examine ratios of observed exit probabilities. Dividing Eq. (5) by $\theta_{i}(0 \mid \tau)$, we get 


$$
\frac{\theta_{i}(1 \mid \tau)}{\theta_{i}(0 \mid \tau)}=\eta_{1 i} \cdot \frac{1-\gamma_{2 i} \theta_{i}(0 \mid \tau-1)-\kappa_{11} \theta_{j}(0 \mid \tau-1)}{1-\theta_{i}(0 \mid \tau-1)-\theta_{j}(0 \mid \tau-1)}
$$

where

$$
\begin{aligned}
\eta_{1 i} & :=\frac{\psi_{1 i}(1)}{\psi_{1 i}(0)}, \quad i=E, N \\
\gamma_{2 i}: & =\frac{\mu_{2 i}}{\mu_{1 i}^{2}}, \quad i=E, N \\
\kappa_{11} & :=\frac{\mu_{1 i 1 j}}{\mu_{1 i} \mu_{1 j}}, \quad i \neq j=E, N .
\end{aligned}
$$

If $v_{N} \equiv v_{E}$ and $\psi_{1 N} \equiv \psi_{1 E}$ then the model determinants of interest can be estimated from a single-risk analysis. Otherwise one has to estimate a bivariate model. We are particularly interested in the relation between $v_{N}$ and $v_{E}$, because this is informative on the validity of the usual assumption that the duration to work is independently right-censored.

If $v_{E} \perp v_{N}$ then $\operatorname{Cov}\left(v_{E}, v_{N}\right)=0$ and $\kappa_{11}=1$. In general, the parameter $\kappa_{11}$ in Eq. (6) is identified from the effect of the past calendar time variation in the exit to destination $j$ on the current exit probability to destination $i$, i.e., on $\theta_{i}(1 \mid \tau), i \neq j$. This fails if $\psi_{2 E}(\tau) \equiv \psi_{2 N}(\tau)$, i.e., if there is no independent variation in calendar time. In this case $\theta_{E}(0 \mid \tau-1) \propto \theta_{N}(0 \mid \tau-1)$, so only $\gamma_{2 i}+$ constant $\kappa_{11}, i=E, N$ are identified from data on $t=0,1$. Thus, identification requires that the exit probabilities from short-term unemployment to nonparticipation and to work do not depend in exactly the same way on calendar time. This can of course be readily examined from time series of $\theta_{E}(0 \mid \tau)$ and $\theta_{N}(0 \mid \tau)$.

The identification condition is reminiscent of the identification condition for continuous-time models mentioned in Sect. 1. If the covariate part of the exit probability to state $i$ does not directly affect the individual exit probability to state $j \neq i$ but does affect the observed exit probability to state $j$ then this indicates that there is a spurious relation between the durations by way of their unobserved determinants. The composition of the survivors at $t=1$ then depends on the speed of the selection process for both exit destinations at $t=0$.

For $t=2$ one can derive similar expressions as above (see Appendix A for a detailed derivation), leading to equations for $\theta_{i}(2 \mid \tau) / \theta_{i}(1 \mid \tau)$ in terms of observables $\theta_{j}(0 \mid \tau-1), \theta_{j}(0 \mid \tau-2), \theta_{j}(1 \mid \tau-1)(j=N, E)$ and additional parameters

$$
\begin{aligned}
\eta_{2 i} & =\frac{\psi_{1 i}(2)}{\psi_{1 i}(1)}, \quad i=E, N \\
\gamma_{3 i} & =\frac{\mu_{3 i}}{\mu_{1 i}^{3}}, \quad i=E, N \\
\kappa_{12} & =\frac{E\left[v_{i} v_{j}^{2}\right]}{\mu_{1 i} \mu_{1 j}^{2}}, \quad \kappa_{21}=\frac{E\left[v_{i}^{2} v_{j}\right]}{\mu_{1 i}^{2} \mu_{1 j}}, \quad i \neq j=E, N
\end{aligned}
$$

which have the same qualitative features as (6). 
We specify $\log \left(\theta_{i}(t \mid \tau) / \theta_{i}(t-1 \mid \tau) \text { ) (with } t=1,2\right)^{3}$ to equal the $\log$ of the corresponding model expression plus an error term. So we estimate a system of four equations. The error terms represent specification errors that are assumed to be identically $N(0, \Sigma)$ distributed over time periods. Note that this is an innocuous assumption because the estimation procedure will lead to consistent estimates even if this assumption were false. In that case, a pseudo-ML estimation procedure should be implemented (see e.g., Gourieroux and Montfort 1996; Wooldridge 2002). We allow the errors in the four equations to be contemporaneously related.

The system is estimated by maximum likelihood (see Appendix C for details). We effectively estimate 11 parameters:

- $\gamma_{2 E}, \gamma_{3 E}, \gamma_{2 N}, \gamma_{3 N}, \kappa_{11}, \kappa_{12}, \kappa_{21}$ are the parameters characterizing the unobserved heterogeneity distribution.

- $\eta_{1 E}, \eta_{2 E}, \eta_{1 N}, \eta_{2 N}$ are the parameters characterizing the pattern of duration dependence in the individual transition probabilities to both destinations during the first two quarters of unemployment.

Note that the duration dependence parameters $\eta_{1 i}$ and $\eta_{2 i}$ are in fact values of the nonparametric function $\psi_{1 i}(t)$. Moreover, the parameters $\gamma_{2 i}, \gamma_{3 i}$ and $\kappa_{j k}$ are the moments of the nonparametrically specified distribution $G$. Note that these moments do not allow for identification of the full heterogeneity distribution. That is to say, in general there will be more than one distribution function $G(v)$ that is consistent with the estimated moments. For more information on recovering a distribution from its estimated moments, see Shohat and Tamarkin (1970) and Lindsay (1989).

Because the heterogeneity distribution is not fully identified, we can only test for uncorrelatedness of the heterogeneity terms, but not for independence. Also note that the parameters describing the heterogeneity distribution appear in all four equations and thus are overidentified.

\section{Data}

We use French administrative data on individuals looking for full time permanent jobs. The French government ${ }^{4}$ kindly provided the data.

\footnotetext{
3 We only take the first two ratios because the model expressions for ratios of higher duration classes quickly become difficult to handle. Second, data for higher duration classes usually are less reliable. This holds a fortiori for the ratio of the hazard probabilities. The standard errors may increase due to the fact that the degree of noise in the data on higher duration classes may be larger. This is illustrated for instance by Van den Berg and Van Ours (1996), who show that the estimation results hardly improve by adding higher duration classes. Moreover, we only need two ratios to illustrate the identification condition and to test for correlation.

4 To be precise, the Département de Marché du Travail of the Ministère du Travail, de l'Emploi et de la Formation Professionelle.
} 
The data are aggregated by (quarterly) duration class. In our empirical analysis, we use quarterly data on the exit probabilities out of the first three quarterly duration classes, over the period 1988.3-1994.4. This means that we have 26 observations on the (ratio of the) exit probability to a given destination. Note that for our model to be applicable it is necessary that the frequency at which the data are collected equals the length of the duration class, in this case 3 months.

At this point it is important to stress that these data are unique, unfortunately also with respect to the time period that is covered. However, the current dataset is very well suited for the purpose of this paper, that is to illustrate our estimation method. Moreover, Van den Berg and Van der Klaauw (2001), by combining detailed micro survey data and less detailed aggregate data on unemployment durations in France, show that both data are mutually consistent in many aspects.

To derive the observed exit probabilities from the data, we use two different data sets. The first data set distinguishes unemployment by elapsed duration and gender type. We do not have any further information on individual characteristics. This is a general drawback of administrative data, this type of data not being collected for the purpose of detailed econometric analyses. These stock data enable us to calculate exit probabilities, aggregated over all possible destinations. Let $U(t \mid \tau)$ denote the number of unemployed in duration class $t$ at the end of quarter $\tau$. Then the observed exit probability equals

$$
\theta(t \mid \tau)=\frac{U(t \mid \tau)-U(t+1 \mid \tau+1)}{U(t \mid \tau)}
$$

The second data set contains the number of unemployed that left unemployment in a given quarter, stratified by destination, duration of the past spell of unemployment and gender type. Five possible destinations are distinguished, viz. nonparticipation, expulsion, training, employment and others. Because of the small number of individuals in some of the categories, we group the destinations: employment and training denote the destination employment, while the other three destinations (nonparticipation, expulsion and other) are taken together in the destination nonparticipation. ${ }^{5}$

The observed destination-specific exit probabilities are now calculated in the following way. Denote the percentage of the outflow to destination $i$ during

\footnotetext{
5 One could argue that expulsion could also be included in the employment destination because one of the reasons for expulsion could be that workers have found a job and therefore do not report to the employment office anymore. However, it turns out that over the calendar time period we investigate there are sudden shifts in the number of exits because of expulsion while the number of exits for other reason has similar sudden shifts in the opposite directions. Apparently expulsions are not well-defined and their numbers are subject to administrative interventions. Nevertheless these interventions do not seem to affect the sum of expulsions and other exits. Therefore, combining them in one state seems the most logical choice.
} 
quarter $\tau$ having been unemployed $t$ quarters by $f_{i}(t \mid \tau){ }^{6}$

$$
\theta_{i}(t \mid \tau)=\frac{U(t \mid \tau-1)-U(t+1 \mid \tau)}{U(t \mid \tau-1)} f_{i}(t \mid \tau)
$$

In order to circumvent modeling seasonal effects, we correct the raw data on the exit probabilities for seasonal effects, using the Census X11 filter (see Shiskin et al. 1967). In Appendix B we show that seasonal effects in the inflow are also corrected for by this procedure.

As noted in the previous section, identification of the unobserved heterogeneity distribution fails if $\theta_{E}$ and $\theta_{N}$ are proportional to each other over calendar time. We can check the time series of $\theta_{E}$ and $\theta_{N}$ to see whether there is evidence for this or not. Figure 1 shows the time series of both $\theta$ 's for males. Clearly, there is independent variation in the movements of the two series over time. To shed some light on the extent of linear dependence, we calculate correlation coefficients. For males (females) the correlation between $\theta_{E}(0 \mid \tau)$ and $\theta_{N}(0 \mid \tau)$ is 0.92 (0.86). Not surprisingly, these correlations are quite high. ${ }^{7}$ This is confirmed by the estimation results below, which show that the estimates of the unobserved heterogeneity parameters have large standard errors. ${ }^{8}$ So the variation in the exit rates allows us to identify the unobserved heterogeneity distribution, but at the same time this variation is such that the precision of the estimates is limited.

\section{Estimation results}

The estimation results are shown in Table 1 . The exit rate to employment shows negative duration dependence during the second quarter of unemployment for both males and females. Moreover, for males, the transition rate to nonparticipation increases during the second quarter of unemployment. The other duration dependence parameters do not differ significantly from one.

The estimates of the unobserved heterogeneity distribution parameters are characterized by large standard errors. The estimates of $\kappa_{11}$ indicate that the unobserved heterogeneity distributions are uncorrelated, for both gender types. If there is independence, the following restrictions should hold:

$$
\kappa_{11}=1, \quad \kappa_{12}=\gamma_{2 N}, \quad \kappa_{21}=\gamma_{2 E} .
$$

\footnotetext{
6 An alternative approach is to divide the observed flows in the second data set by an appropriate risk set, based on the stocks in the first data set. The risk set for the flow from class $t$ in quarter $\tau$ is of the form $\alpha U(t \mid \tau-1)+\beta U(t-1 \mid \tau-1)$, with $\alpha+\beta=1$. For reasonable values of $\alpha$ and $\beta$, this gives similar estimation results.

7 The high correlations are not caused by the correction for seasonal effects. The unadjusted data also show high correlations.

8 Inference on $\gamma_{3 E}$ and $\gamma_{3 N}$ is troublesome because the observed time patterns of $\theta_{E}(0 \mid \tau-$ 2) $\theta_{N}(1 \mid \tau-1)+\theta_{N}(0 \mid \tau-2) \theta_{E}(1 \mid \tau-1)$ and $\theta_{E}(0 \mid \tau-2) \theta_{E}(1 \mid \tau-1)$ are almost proportional.
} 
Table 1 Parameter estimates

\begin{tabular}{|c|c|c|c|c|c|c|c|c|}
\hline & \multicolumn{4}{|c|}{ Unrestricted model } & \multicolumn{4}{|c|}{ Restricted model } \\
\hline & \multicolumn{2}{|l|}{ Males } & \multicolumn{2}{|c|}{ Females } & \multicolumn{2}{|l|}{ Males } & \multicolumn{2}{|c|}{ Females } \\
\hline \multicolumn{9}{|c|}{ Unobserved heterogeneity distribution } \\
\hline$\gamma_{2 E}$ & 1.372 & $(0.137)$ & 1.324 & $(0.148)$ & 1.446 & $(0.070)$ & 1.519 & $(0.098)$ \\
\hline$\gamma_{3 E}$ & 1.002 & $(0.079)$ & 1.005 & $(0.167)$ & 2.030 & $(0.262)$ & 2.170 & $(0.469)$ \\
\hline$\gamma_{2 N}$ & 1.254 & $(0.314)$ & 1.215 & $(0.242)$ & 1.432 & $(0.129)$ & 1.570 & $(0.127)$ \\
\hline$\gamma_{3 N}$ & 1.005 & $(0.189)$ & 1.007 & $(0.335)$ & 1.430 & $(0.650)$ & 1.793 & $(0.867)$ \\
\hline$\kappa_{11}$ & 1.120 & $(0.192)$ & 1.284 & $(0.167)$ & & & & \\
\hline$\kappa_{12}$ & 0.939 & $(0.572)$ & 1.368 & $(0.702)$ & & & & \\
\hline$\kappa_{21}$ & 2.372 & $(0.429)$ & 2.423 & $(0.574)$ & & & & \\
\hline \multicolumn{9}{|c|}{ Duration dependence } \\
\hline$\eta_{1 E}$ & 1.053 & $(0.037)$ & 1.058 & $(0.032)$ & 1.041 & $(0.028)$ & 1.047 & $(0.032)$ \\
\hline$\eta_{2 E}$ & 0.929 & $(0.031)$ & 0.938 & $(0.028)$ & 0.928 & $(0.023)$ & 0.932 & $(0.028)$ \\
\hline$\eta_{1 N}$ & 0.907 & $(0.041)$ & 0.985 & $(0.030)$ & 0.918 & $(0.037)$ & 0.986 & $(0.030)$ \\
\hline$\eta_{2 N}$ & 0.991 & $(0.048)$ & 1.024 & $(0.037)$ & 0.990 & $(0.038)$ & 1.028 & $(0.037)$ \\
\hline \multicolumn{9}{|c|}{ Log-likelihood } \\
\hline & \multicolumn{2}{|c|}{331.71} & \multicolumn{2}{|c|}{351.49} & \multicolumn{2}{|c|}{331.22} & \multicolumn{2}{|c|}{350.20} \\
\hline
\end{tabular}

Standard errors in parentheses

Table 1 also shows the estimation results of the model in which these restrictions are imposed. The likelihood ratio test statistic equals 0.98 for males and 2.58 for females. As the $10 \%$-critical value of the $\chi^{2}(3)$ distribution is 6.25 , we do not reject the independence assumption at any reasonable significance level. The parameter estimates of the duration dependence parameters are close to the estimates in the unrestricted model. Duration dependence in the exit rate to employment is similar for males and females, with significant negative duration dependence during the second quarter of unemployment.

Concerning the unobserved heterogeneity parameters, note that a $\gamma_{2 i}$ estimate significantly larger than one implies that $\operatorname{var}\left(v_{i}\right)>0$, i.e., that unobserved heterogeneity is present. For both males and females, we find unobserved heterogeneity in the exit rate to both employment and nonparticipation, which is hardly surprising, given the high level of aggregation in the data. Further, $\gamma_{3 i}-\gamma_{2 i}^{2}$ does not differ significantly from zero, for each gender. From Shohat and Tamarkin (1970), this implies that the marginal distributions of $v_{i}$ can be accurately described by a discrete distribution with one positive point of support and one point of support equal to zero. This is convenient for the interpretation of the results, for two reasons. First, for bivariate discrete distributions with two by two points of support, uncorrelatedness is equivalent to independence. Secondly, it is easy to summarize the bivariate distribution in words. There is not much difference across gender. About two third has a high probability of finding a job, while one third never finds job. ${ }^{9}$ The probability of becoming

\footnotetext{
9 Remember that the model assumes that the unobserved heterogeneity of an individual does not change over time. By taking two points of support, we implicitly divide the group of unemployed individuals in two categories, each category characterized by the value of the unobserved heterogeneity parameter $v_{E}$. It turns out that one category of unemployed has a value equal to 0 . People in this category therefore will never find a job.
} 
nonparticipant is positive for almost all unemployed. This makes sense, given the fact that one can personally decide to become nonparticipant.

\section{Conclusion}

The individual conditional exit probabilities to employment and nonparticipation are uncorrelated across individuals, for males as well as females. This suggests that one may treat exits to nonparticipation as independent right-censoring of the duration until work, at least under the current set of covariates. These results do not depend on arbitrary functional-form assumptions concerning duration dependence or the unobserved heterogeneity distribution. Moreover, we find that the duration dependence patterns and heterogeneity distributions of the conditional exit probabilities to employment and nonparticipation are different from each other. The exit to employment displays negative duration dependence after one quarter of unemployment, while the exit to nonparticipation does not display duration dependence for females, and negative duration dependence during the first quarter for males. Therefore, in the outflow from unemployment, it is important to distinguish between exits to employment and exits to nonparticipation. The differences in dynamics between the two transitions have to be taken into account.

Our model and estimation method need discrete-time time-series data on aggregate numbers of individuals in different unemployment duration classes. Such aggregate data have advantages in terms of calendar time span and lack of attrition. But, the main disadvantage is the lack of detail. A topic for future research is a comparison of our approach with methods designed and implemented for smaller samples of detailed micro data. ${ }^{10}$

\section{Appendices}

\section{A Model expressions for higher duration classes}

In this appendix we derive the model expression for

$$
\frac{\theta_{i}(2 \mid \tau)}{\theta_{i}(1 \mid \tau)}, \quad i=E, N
$$

Considering the third duration class, we know that

$$
\begin{aligned}
\operatorname{Pr}(t \geq 2 \mid \tau) & =(1-\operatorname{Pr}(t=0 \mid \tau-2)) \cdot(1-\operatorname{Pr}(t=1 \mid \tau-1, t \geq 1)) \\
& =\left(1-\theta_{E}(0 \mid \tau-2)-\theta_{N}(0 \mid \tau-2)\right) \times\left(1-\theta_{E}(1 \mid \tau-1)-\theta_{N}(1 \mid \tau-1)\right)
\end{aligned}
$$

10 See e.g., Fermanian (2003) for a Kernel-based estimation method for a class of competing risks models larger than the continuous-time MPH model. 


$$
\begin{aligned}
\operatorname{Pr}(t=2 \wedge E \mid \tau)= & E_{v_{E}, v_{N}}\left[\left(1-\psi_{1 E}(0) \psi_{2 E}(\tau-2) v_{E}-\psi_{1 N}(0) \psi_{2 N}(\tau-2) v_{N}\right)\right. \\
& \times\left(1-\psi_{1 E}(1) \psi_{2 E}(\tau-1) v_{E}-\psi_{1 N}(1) \psi_{2 N}(\tau-1) v_{N}\right) \\
& \left.\times \psi_{1 E}(2) \psi_{2 E}(\tau) v_{E}\right]
\end{aligned}
$$

Using the expression for $\theta_{E}(1 \mid \tau)$ in Eq. (5), we get

$$
\begin{aligned}
\frac{\theta_{E}(2 \mid \tau)}{\theta_{E}(1 \mid \tau)}= & \eta_{2 E} \cdot \frac{c_{E}(\tau)}{1-\theta_{E}(0 \mid \tau-1) \gamma_{2 E}-\theta_{N}(0 \mid \tau-1) \kappa_{11}} \\
& \times\left[1-\left(\theta_{E}(1 \mid \tau-1)+\theta_{E}(0 \mid \tau-2)\right) \gamma_{2 E}+\theta_{E}(0 \mid \tau-2) \theta_{E}(1 \mid \tau-1) \gamma_{3 E}\right. \\
& -\left(\theta_{N}(1 \mid \tau-1)+\theta_{N}(0 \mid \tau-2)\right) \kappa_{11}+\theta_{N}(0 \mid \tau-2) \theta_{N}(1 \mid \tau-1) \kappa_{12} \\
& \left.+\left(\theta_{E}(0 \mid \tau-2) \theta_{N}(1 \mid \tau-1)+\theta_{N}(0 \mid \tau-2) \theta_{E}(1 \mid \tau-1)\right) \kappa_{21}\right]
\end{aligned}
$$

where

$$
\begin{aligned}
c_{i}(\tau) & =\frac{1-\theta_{i}(0 \mid \tau-1)-\theta_{j}(0 \mid \tau-1)}{\left(1-\theta_{i}(0 \mid \tau-2)-\theta_{j}(0 \mid \tau-2)\right)\left(1-\theta_{i}(1 \mid \tau-1)-\theta_{j}(1 \mid \tau-1)\right)}, \quad i \neq j=E, N \\
\eta_{2 i} & =\frac{\psi_{1 i}(2)}{\psi_{1 i}(1)}, \quad i=E, N \\
\gamma_{3 i} & =\frac{\mu_{3 i}}{\mu_{1 i}^{3}, \quad i=E, N} \\
\kappa_{12} & =\frac{E\left[v_{E} v_{N}^{2}\right]}{\mu_{1 E} \mu_{1 N}^{2}}, \quad \kappa_{21}=\frac{E\left[v_{E}^{2} v_{N}\right]}{\mu_{1 E}^{2} \mu_{1 N}} .
\end{aligned}
$$

Finally, the second ratio equation for exit to $N$ equals:

$$
\begin{aligned}
\frac{\theta_{N}(2 \mid \tau)}{\theta_{N}(1 \mid \tau)}= & \eta_{2 N} \cdot \frac{c_{N}(\tau)}{1-\theta_{N}(0 \mid \tau-1) \gamma_{2 N}-\theta_{E}(0 \mid \tau-1) \kappa_{11}} \\
& \times\left[1-\left(\theta_{N}(1 \mid \tau-1)+\theta_{N}(0 \mid \tau-2)\right) \gamma_{2 N}+\theta_{N}(0 \mid \tau-2) \theta_{N}(1 \mid \tau-1) \gamma_{3 N}\right. \\
& -\left(\theta_{E}(1 \mid \tau-1)+\theta_{E}(0 \mid \tau-2)\right) \kappa_{11}+\theta_{E}(0 \mid \tau-2) \theta_{E}(1 \mid \tau-1) \kappa_{21} \\
& \left.+\left(\theta_{N}(0 \mid \tau-2) \theta_{E}(1 \mid \tau-1)+\theta_{E}(0 \mid \tau-2) \theta_{N}(1 \mid \tau-1)\right) \kappa_{12}\right]
\end{aligned}
$$

where $c_{N}(\tau), \eta_{2 N}, \gamma_{2 N}, \gamma_{3 N}, \kappa_{12}$, and $\kappa_{21}$ are defined above.

\section{B Seasonal adjustment}

In this appendix we show that adjusting the data for seasonal effects also eliminates seasonal effects in the inflow. The way we proceed is as follows: we first derive the ratio equations when we allow for seasonal effects in the inflow. We 
then estimate the modified model, showing this extra term to be non significant. We assume the seasonal effects in the inflow to act by extra term in the MPH specification of the exit probabilities. This method is observationally equivalent to making the unobserved heterogeneity distribution $G$ dependent on $\tau$ (See Abbring et al. 2002). The exit probability to destination $i$ after $t$ periods of unemployment, conditionally given the explanatory variables $\tau$ and $v_{i},(i=E, N)$, then equals

$$
\begin{aligned}
& \theta_{E}\left(t \mid \tau, v_{E}\right)=\psi_{1 E}(t) \psi_{2 E}(\tau) \psi_{3 E}(\tau-t) v_{E}, \\
& \theta_{N}\left(t \mid \tau, v_{N}\right)=\psi_{1 N}(t) \psi_{2 N}(\tau) \psi_{3 N}(\tau-t) v_{N} .
\end{aligned}
$$

Applying the same method as in Sect. 2.2 gives the following ratio equation:

$$
\begin{aligned}
& \frac{\theta_{E}(1 \mid \tau)}{\theta_{E}(0 \mid \tau)}=\eta_{1 E} \cdot \frac{\psi_{3 E}(\tau-1)}{\psi_{3 E}(\tau)} \cdot \frac{1-\gamma_{2 E} \theta_{E}(0 \mid \tau-1)-\kappa_{11} \theta_{N}(0 \mid \tau-1)}{1-\theta_{E}(0 \mid \tau-1)-\theta_{N}(0 \mid \tau-1)} \\
& \frac{\theta_{N}(1 \mid \tau)}{\theta_{N}(0 \mid \tau)}=\eta_{1 N} \cdot \frac{\psi_{3 N}(\tau-1)}{\psi_{3 N}(\tau)} \cdot \frac{1-\gamma_{2 N} \theta_{N}(0 \mid \tau-1)-\kappa_{11} \theta_{E}(0 \mid \tau-1)}{1-\theta_{N}(0 \mid \tau-1)-\theta_{E}(0 \mid \tau-1)} .
\end{aligned}
$$

For estimation, the fraction $\frac{\psi_{3 i}(\tau-1)}{\psi_{3 i}(\tau)}$ is parameterized as follows:

$$
\begin{aligned}
\psi_{3 i}(\mathrm{IV}) & =\omega_{1 i} \psi_{3 i}(\mathrm{I}) \\
\psi_{3 i}(\mathrm{I}) & =\omega_{2 i} \psi_{3 i}(\mathrm{II}) \\
\psi_{3 i}(\mathrm{II}) & =\omega_{3 i} \psi_{3 i}(\mathrm{III}) \\
\psi_{3 i}(\mathrm{III}) & =\frac{1}{\omega_{1 i} \cdot \omega_{2 i} \cdot \omega_{3 i}} \psi_{3 i}(\mathrm{IV})
\end{aligned}
$$

We now estimate the extended model as described in Sect. 2.2. The extra parameters to be estimated then are $\ln \left(\omega_{1 i}\right), \ln \left(\omega_{2 i}\right), \ln \left(\omega_{3 i}\right)$. We only estimate the first ratio equation because if the seasonal adjustment does not correct for seasonal effects in the inflow, the first ratio equation suffices to show this. This has the advantage that we do not have to estimate the poorly identified parameters. Without loss of generality we assume the heterogeneity distributions to be independent (i.e., $\kappa_{11}=1$ ). Table 2 shows the estimation results.

Testing for the hypothesis that adjusting for seasonal effects also corrects for seasonal effects in the inflow is equivalent to testing the hypothesis

$$
\forall i: \ln \left(\omega_{1 i}\right)=\ln \left(\omega_{2 i}\right)=\ln \left(\omega_{3 i}\right)=0 .
$$

From the estimation results it follows that we cannot reject this hypothesis at any reasonable significance level, as the $10 \%$-critical value of the $\chi^{2}(6)$-distribution equals 10.64 . 
Table 2 Parameter estimates

\begin{tabular}{|c|c|c|c|c|c|c|c|c|}
\hline & \multicolumn{4}{|l|}{ Males } & \multicolumn{4}{|c|}{ Females } \\
\hline \multicolumn{9}{|c|}{ Employment } \\
\hline$\eta_{1 E}$ & 1.053 & $(0.030)$ & 1.053 & $(0.030)$ & 1.076 & $(0.034)$ & 1.078 & $(0.032)$ \\
\hline$\gamma_{2 E}$ & 1.479 & $(0.073)$ & 1.479 & $(0.073)$ & 1.614 & $(0.098)$ & 1.619 & $(0.092)$ \\
\hline $\ln \left(\omega_{1 E}\right)$ & & & 0.005 & $(0.012)$ & & & 0.004 & $(0.009)$ \\
\hline $\ln \left(\omega_{2 E}\right)$ & & & -0.004 & $(0.012)$ & & & 0.007 & $(0.009)$ \\
\hline $\ln \left(\omega_{3 E}\right)$ & & & 0.002 & $(0.012)$ & & & 0.005 & (0.009) \\
\hline \multicolumn{9}{|c|}{ Nonparticipation } \\
\hline$\eta_{1 N}$ & 0.905 & $(0.036)$ & 0.905 & $(0.036)$ & 0.990 & $(0.031)$ & 0.989 & $(0.030)$ \\
\hline$\gamma_{2 N}$ & 1.386 & $(0.130)$ & 1.383 & $(0.130)$ & 1.592 & $(0.128)$ & 1.588 & $(0.125)$ \\
\hline $\ln \left(\omega_{1 N}\right)$ & & & -0.001 & $(0.012)$ & & & -0.005 & $(0.008)$ \\
\hline $\ln \left(\omega_{2 N}\right)$ & & & 0.004 & $(0.012)$ & & & 0.005 & $(0.008)$ \\
\hline $\ln \left(\omega_{3 N}\right)$ & & & 0.002 & $(0.012)$ & & & 0.001 & $(0.008)$ \\
\hline \multicolumn{9}{|c|}{ Log-likelihood } \\
\hline & \multicolumn{2}{|c|}{170.22} & \multicolumn{2}{|c|}{170.48} & \multicolumn{2}{|c|}{185.35} & \multicolumn{2}{|c|}{187.47} \\
\hline
\end{tabular}

Standard errors in parentheses

\section{Likelihood function}

Using maximum likelihood we estimate the following system of equations:

$$
\begin{aligned}
\log \left(\frac{\theta_{E}(1 \mid \tau)}{\theta_{E}(0 \mid \tau)}\right)= & \log \left(\eta_{1 E} \cdot \frac{1-\gamma_{2 E} \theta_{E}(0 \mid \tau-1)-\kappa_{11} \theta_{N}(0 \mid \tau-1)}{1-\theta_{E}(0 \mid \tau-1)-\theta_{N}(0 \mid \tau-1)}\right)+\epsilon_{1 E} \\
\log \left(\frac{\theta_{N}(1 \mid \tau)}{\theta_{N}(0 \mid \tau)}\right)= & \log \left(\eta_{1 N} \cdot \frac{1-\gamma_{2 N} \theta_{N}(0 \mid \tau-1)-\kappa_{11} \theta_{E}(0 \mid \tau-1)}{1-\theta_{N}(0 \mid \tau-1)-\theta_{E}(0 \mid \tau-1)}\right)+\epsilon_{1 N} \\
\log \left(\frac{\theta_{E}(2 \mid \tau)}{\theta_{E}(1 \mid \tau)}\right)= & \log \left(\eta_{2 E} \cdot \frac{c_{E}(\tau)}{1-\theta_{E}(0 \mid \tau-1) \gamma_{2 E}-\theta_{N}(0 \mid \tau-1) \kappa_{11}}\right. \\
& \times\left[1-\left(\theta_{E}(1 \mid \tau-1)+\theta_{E}(0 \mid \tau-2)\right) \gamma_{2 E}+\theta_{E}(0 \mid \tau-2) \theta_{E}(1 \mid \tau-1) \gamma_{3 E}\right. \\
& -\left(\theta_{N}(1 \mid \tau-1)+\theta_{N}(0 \mid \tau-2)\right) \kappa_{11}+\theta_{N}(0 \mid \tau-2) \theta_{N}(1 \mid \tau-1) \kappa_{12} \\
& \left.\left.+\left(\theta_{E}(0 \mid \tau-2) \theta_{N}(1 \mid \tau-1)+\theta_{N}(0 \mid \tau-2) \theta_{E}(1 \mid \tau-1)\right) \kappa_{21}\right]\right)+\epsilon_{2 E}
\end{aligned}
$$

$$
\begin{aligned}
\log \left(\frac{\theta_{N}(2 \mid \tau)}{\theta_{N}(1 \mid \tau)}\right)= & \log \left(\eta_{2 N} \cdot \frac{c_{N}(\tau)}{1-\theta_{N}(0 \mid \tau-1) \gamma_{2 N}-\theta_{E}(0 \mid \tau-1) \kappa_{11}}\right. \\
& \times\left[1-\left(\theta_{N}(1 \mid \tau-1)+\theta_{N}(0 \mid \tau-2)\right) \gamma_{2 N}+\theta_{N}(0 \mid \tau-2) \theta_{N}(1 \mid \tau-1) \gamma_{3 N}\right. \\
& -\left(\theta_{E}(1 \mid \tau-1)+\theta_{E}(0 \mid \tau-2)\right) \kappa_{11}+\theta_{E}(0 \mid \tau-2) \theta_{E}(1 \mid \tau-1) \kappa_{21} \\
& \left.\left.+\left(\theta_{N}(0 \mid \tau-2) \theta_{E}(1 \mid \tau-1)+\theta_{E}(0 \mid \tau-2) \theta_{N}(1 \mid \tau-1)\right) \kappa_{12}\right]\right)+\epsilon_{2 N}
\end{aligned}
$$


with

$$
\left[\begin{array}{l}
\epsilon_{1 E} \\
\epsilon_{1 N} \\
\epsilon_{2 E} \\
\epsilon_{2 N}
\end{array}\right] \sim N(0, \Sigma) .
$$

\section{References}

Abbring JH, Van den Berg GJ (2003) The identifiability of the mixed proportional hazards competing risks model. J R Stat Soc Ser B 65:701-710

Abbring JH, Van den Berg GJ, Van Ours JC (2002) The anatomy of unemployment dynamics. Eur Econ Rev 46:1785-1824

Fermanian JD (2003) Nonparametric estimation of competing risks models with covariates. J Multivariate Anal 85:156-191

Flinn CJ, Heckman JJ (1983) Are unemployment and out of the labor force behaviorally distinct labor force states. J Labor Econ 1:28-42

Gourieroux C, Montfort A (1996) Simulation-based econometric methods. Oxford University Press, Oxford

Heckman JJ, Honoré BE (1989) The identifiability of the competing risks model. Biometrika 76:325-330

Lancaster T (1990) The econometric analysis of transition data. Cambridge University Press, Cambridge

Lindsay BG (1989) Moment matrices: applications in mixtures. Ann Stat 17:722-740

Manning A, Machin S (1999) The causes and consequences of long-term unemployment in Europe. In: Ashenfelter O, Card D (eds) Handbook of labor economics, vol 3. North Holland, Amsterdam

Shiskin J, Young AH, Musgrave JC (1967) The X-11 variant of the census method II seasonal adjustment program. Technical paper 15, Bureau of the Census, US Department of Commerce

Shohat JA, Tamarkin JD (1970) The problem of moments. American Mathematical Society, Providence

Van den Berg GJ (2001) Duration models: specification, identification, and multiple durations. In: Heckman JJ, Leamer E (eds) Handbook of econometrics, vol V. North Holland, Amsterdam

Van den Berg GJ, Van Ours JC (1996) Unemployment dynamics and duration dependence. J Labor Econ 14:100-125

Van den Berg GJ, Van der Klaauw B (2001) Combining micro and macro unemployment duration data. J Econom 102:271-309

Wooldridge JM (2002) Econometric analysis of cross-section and panel data. MIT Press, Cambridge 